International Journal of Electrical and Power Engineering 5 (1): 24-27, 2011

ISSN: 1990-7958

(C) Medwell Journals, 2011

\title{
Modeling of a 6H-SiC MESFET for High-Power and High-Gain Applications
}

\author{
H. Arabshahi and M. Rezaee Rokn-Abadi \\ Department of Physics, Ferdowsi University of Mashhad, Mashhad, Iran
}

\begin{abstract}
A Monte Carlo simulation has been used to model steady state and transient electron transport in $6 \mathrm{H}-\mathrm{SiC}$ field effect transistor. The simulated device geometries and doping are matched to the nominal parameters described for the experimental structures as closely as possible and the predicted 1-5 and transfer characteristics for the intrinsic devices show fair agreement with the available experimental data. Simulations of the effect of modulating, the gate bias have also been carried out to test the device response and derived the frequency bandwidth. Value of $90 \pm 10 \mathrm{GHz}$ has been derived for the intrinsic current gain cut-off frequency of the $6 \mathrm{H}-\mathrm{SiC}$ MESFETs.
\end{abstract}

Key words: Steady-state, transient, cut-off frequency, frequency bandwidth, geometries, $6 \mathrm{H}-\mathrm{SiC}$

\section{INTRODUCTION}

$6 \mathrm{H}-\mathrm{SiC}$ is a wide band gap semiconductor $\left(\mathrm{E}_{\mathrm{g}}=3 \mathrm{eV}\right)$ and therefore has a high breakdown field and low thermal generation rate. These properties combined with good thermal conductivity and stability make $6 \mathrm{H}-\mathrm{SiC}$ an attractive material for high power, high temperature and radiation harsh environment electronic devices. Monte Carlo simulations predict a peak electron velocity of $3 \times 10^{7} \mathrm{~cm} \mathrm{sec}^{-1}$ and a saturation electron velocity of $1.3 \times 10^{7} \mathrm{~cm} \mathrm{sec}^{-1}$ (Mickevicius and Zhao, 1998; Trew and Shin, 1994). This makes possible high frequency operation of $\mathrm{SiC}$ devices. $6 \mathrm{H}-\mathrm{SiC}$ is believed to be the most important $\mathrm{SiC}$ poly-type (in comparison to $3 \mathrm{C}$ or $4 \mathrm{H}-\mathrm{SiC}$ ) for high reliability power field effect transistor technology (Dohnke et al., 1994; Agarwal, 1997; Joshi, 1995; Nilsson et al., 1996) due to the higher than 4H-SiC conduction band offset with $\mathrm{SiO}_{2}$. In this study, we analyze a Monte Carlo simulation which is used to model electron transport in hexagonal $6 \mathrm{H}-\mathrm{SiC}$ MESFETs.

\section{THE SIMULATION MODEL}

Electron particles in the ensemble Monte Carlo simulation occupy non-parabolic ellipsoidal valleys in reciprocal space and obey Boltzmann statistics. Herring-Vogt transformations are used to map carrier momenta into spherical valleys when particles are drifted, scattered or cross heterojunctions (where care has been taken to ensure that the crystal momentum in the plane of the junction is conserved across the interface). The electric field equations are solved self-consistently with the electron transport using a finite difference method
(Walmsley and Abram, 1996) and the device grid potentials are updated at each ensemble drift time step (1 femto sec). Electrons in the bulk are scattered by ionized impurities and by bulk acoustic and non-polar optical phonon modes. Intervalley scattering by the absorption and emission of long wavelength acoustic and optic phonons have also been considered in the model. Model devices are built up as a series of joined rectangular regions with the electric field cell sizes matched along the join between each region (Arabshahi, 2006; Arabshahi and Ghasemian, 2006). Each region can consist of multiple layers of different alloy composition and doping/compensation density. The 6H-SiC MESFET can be described simply by three regions (Fig. 1), representing source and drain doping implants and a central region containing the supply layers. The field cell size used for the central region is $30 \mathrm{~nm}^{2}$ (horizontal $\mathrm{x}$ vertical) but that in the high doped source and drain implants is finer $\left(10 \mathrm{~nm}^{2}\right)$.

Simulations of steady current characteristics have been carried out using 20000 electron particles; later simulations to derive the frequency response have required 50000 particles. Figure 1 shows a schematic of the modeled $6 \mathrm{H}-\mathrm{SiC}$ MESFET. The overall device length is $2 \mu \mathrm{m}$ in the $\mathrm{x}$-direction and the device has a $0.2 \mu \mathrm{m}$ gate length and $0.15 \mu \mathrm{m}$ source and drain length. The source and drain have Ohmic contacts and gate is in Schottky contact in $1 \mathrm{eV}$ to represent the contact potential at $\mathrm{Au} / \mathrm{Pt}$. The source and drain regions are doped to $2 \times 10^{24} \mathrm{~m}^{-3}$ electron concentration and the top and down buffer layers are doped to $2 \times 10^{23} \mathrm{~m}^{-3}$ and $5 \times 10^{22} \mathrm{~m}^{-3}$ electron concentration, respectively. Subsequent to modeling the steady state characteristics, simulations of the effect of

Corresponding Author: H. Arabshahi, Department of Physics, Ferdowsi University of Mashhad, Mashhad, Iran 
modulating the gate bias have also been carried out in order to test the drain current response and drive the cut-off frequency corresponding to unit current gain. The frequency response was investigated by applying truncated sinc voltage pulse to both gate contacts of the $6 \mathrm{H}-\mathrm{SiCMESFET}$. The time dependence of the voltage signal is:

$$
\mathrm{V}_{\text {gate }}(\mathrm{t})=\mathrm{V}_{0}+\mathrm{V}_{\text {amplitite }} \times \frac{\sin \omega(\mathrm{t}-\mathrm{T} / 2)}{\omega(\mathrm{t}-\mathrm{T} / 2)}
$$

With:

Where:

$$
\omega=2 \pi \mathrm{T} / \mathrm{T}_{\operatorname{mini}}
$$

$\mathrm{V}_{0} \quad=$ Steady gate bias upon which the modulation signal is superimposed

$\mathrm{V}_{\text {amplitude }}=$ Peak voltage during the signal

$\mathrm{T}=$ Duration of the signal

$\mathrm{T}_{\text {mini }}=$ Duration of each minicycle within that signal

Table 1: Important parameters used in the simulations for $6 \mathrm{H}-\mathrm{SiC}$ material Parameters $6 \mathrm{H}-\mathrm{SiC}$

Density $\rho\left(\mathrm{kgm}^{-3}\right)$ 3200

Longitudinal sound velocity $\mathrm{v}^{6}\left(\mathrm{~ms}^{-1}\right)$

Low-frequency dielectric constant $\varepsilon^{s}$

1373

High-frequency dielectric constant $\varepsilon_{\text {eo }}$

9.7

Acoustic deformation potential (eV)

Polar optical phonon energy $(\mathrm{eV})$

$\Gamma$-valley effective mass $\left(\mathrm{m}^{*}\right)$

$\underline{\Gamma \text {-valley nonparabolicity }\left(\mathrm{eV}^{-1}\right)}$

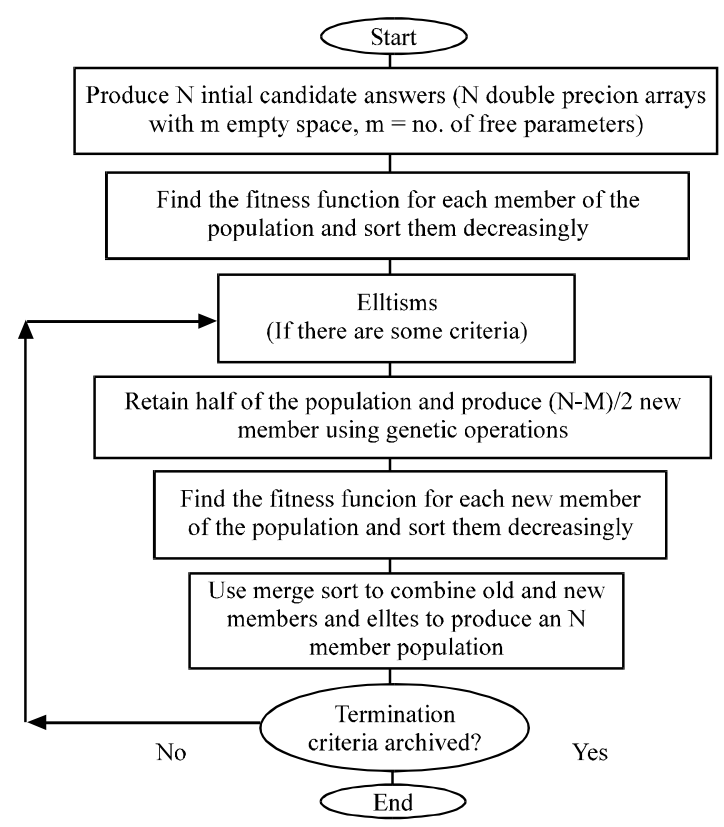

Fig. 1: The 2-dimensional model of $6 \mathrm{H}$-SiC MESFET. The modeled structure is divided into three regions as indicated. Electron particles are initially distributed keeping all regions charge neutral. The location of the source and the drain implants and the top and back buffer layer are marked
In this instance 10 minicycles have been used. A 100 ps duration sinc-form pulse containing 10 minicycles has the specific advantage of providing a flat frequency spectrum up to $100 \mathrm{GHz}$ with $10 \mathrm{GHz}$ resolution. The material parameters used in the simulation are shown in Table 1.

\section{SIMULATION RESULTS}

Figure 2 shows the simulated drain current-voltage characteristic for the $6 \mathrm{H}-\mathrm{SiC}$ MESFET with the gate voltage descending from -1 to $-13 \mathrm{~V}$ in -2 intervals. The simulated characteristics at room temperature show good saturation behavior with a knee voltage around $20-30 \mathrm{~V}$ and a saturation drain current of about $1450 \mathrm{~mA} \mathrm{~mm}^{-1}$ for $\mathrm{V}_{\mathrm{gs}}=-1 \mathrm{~V}$. The high drain current density is encouraging for the use of $6 \mathrm{H}-\mathrm{SiC}$ for high power applications. It is also clear that the device is not completely pinched-off even at large negative gate bias $\left(V_{g s}=-13 \mathrm{~V}\right)$ which is due to strong electron injection into the buffer layer at high electric fields.

An increasing fraction of the drain current flows through the buffer as the drain voltage increases. Figure 3 and 4, respectively show the steady state $\Gamma$-valley band profile and the total electron density as a function of distance from the source when the drainsource potential drop is $20 \mathrm{~V}$ and the gate voltage is $-1 \mathrm{~V}$. Almost all the drain-source potential is dropped within the gate-drain region of the channel, leaving a flat potential profile near the source and drain. As electrons move towards the drain, they lose potential energy and gain sufficient kinetic energy to transfer to the upper conduction valleys where their drift velocity is reduced. Figure 4 shows the electron density through the device. The gate depletion region is clearly seen where the

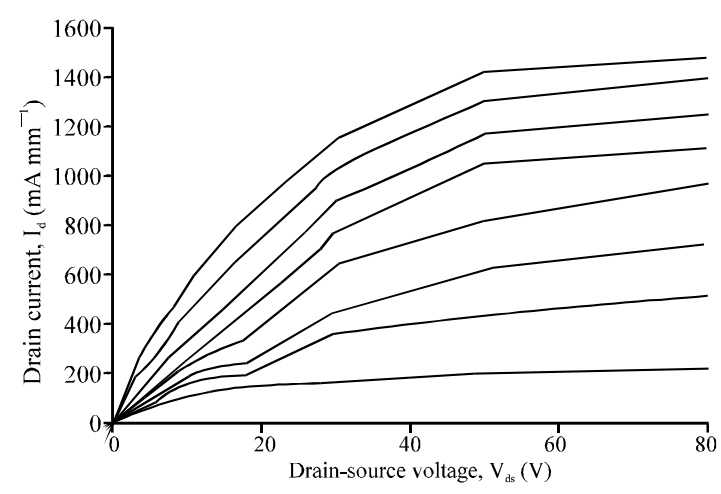

Fig. 2: Simulated current-voltage characteristics for the $6 \mathrm{H}-\mathrm{SiC}$ MESFET at $300 \mathrm{~K}$. The gate voltage ranges from -1 to $-13 \mathrm{~V}$ in $-2 \mathrm{~V}$ intervals. The knee voltage at around $20-30 \mathrm{~V}$ 


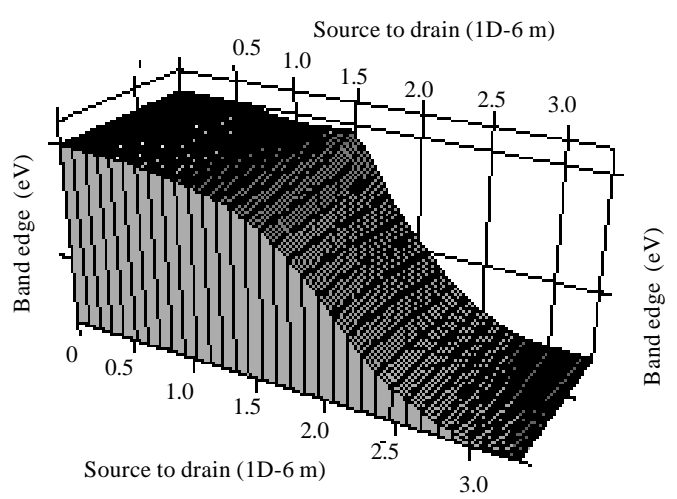

Fig. 3: Interpolated contour plot showing the steady state $\Gamma$-valley band profile through the simulated $6 \mathrm{H}-\mathrm{SiC}$ MESFET at room temperature when the bias applied to the gate is $-1 \mathrm{~V}$ and the drainsource bias is $20 \mathrm{~V}$

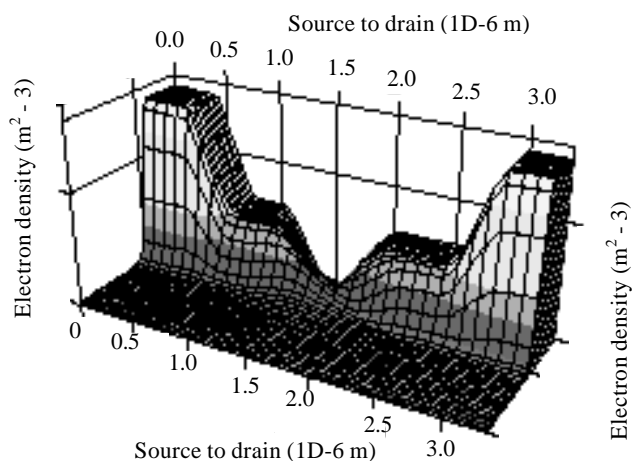

Fig. 4: Logarithmic plot indicating the electron density throughout the modeled region of the $6 \mathrm{H}-\mathrm{SiC}$ MESFET when the bias applied to the gate is $-1 \mathrm{~V}$ and the drain-source bias is $20 \mathrm{~V}$

electron density is several orders of magnitude lower than it is near the source and drain. Figure 5a-c, respectively shows the sinc voltage signal applied to the gate, the drain current response and the gate current for the simulated 6H-SiC MESFET.

In Fig. 5a-c, the gate current $\left(\mathrm{I}_{\mathrm{gtt}}\right)$ is electric displacement, so resembles the derivative of the sinc voltage $\left(\mathrm{V}_{\text {gate }}\right)$. The gate current shown is the sum of the electric displacement, occurring at the left and right hand electrodes (Fig. 1).

The reproduction of the sinc pulse in the time dependence of the drain current is easy to discern, despite high frequency noise on the recorded currents which is a direct consequence of the motion of the finite number of electron particles across the field cell grid in the simulation. The current gain has been derived as a function of frequency by taking fast Fourier

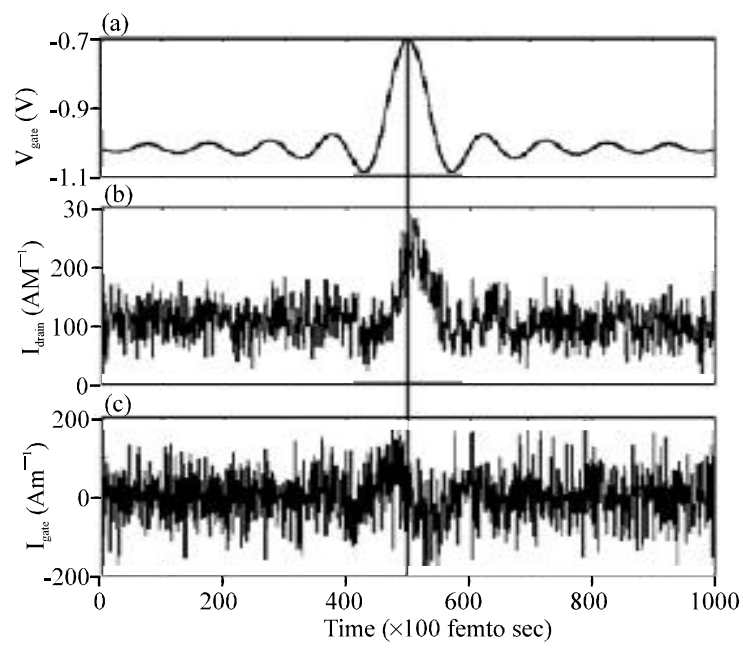

Fig. 5: The simulated frequency response of $6 \mathrm{H}-\mathrm{SiC}$ MESFET to a sinc gate voltage pulse. From top to bottom, this figure shows the gate voltage $\left(\mathrm{V}_{\text {gate }}\right)$ as a function of time for a truncated pulse of duration $100 \mathrm{ps}$, the drain current response $\left(\mathrm{I}_{\text {drain }}\right)$ and the gate current $\left(\mathrm{I}_{\text {gate }}\right)$ which is electric displacement

transforms of the simulated drain and gate current signals then obtaining the ratios of the coefficients of the drain and gate current transforms. The calculated cut-off frequency for the simulated device is about $90 \pm 10 \mathrm{GHz}$. If the data are extrapolated to the low frequency limit, the current gain is expected to be of the order of $20 \mathrm{~dB}$.

\section{CONCLUSION}

A Monte Carlo simulation was used to model steady state and transient electron transport in a $6 \mathrm{H}-\mathrm{SiC}$ metal semiconductor field effect transistor. The simulation results show that due to the high drain current density we can expect $6 \mathrm{H}-\mathrm{SiC}$ devices have superior high power and high gain performance. The frequency response of the device has also been studied by applying a sinc pulse and sinusoidal signals at a range of frequencies to the gate.

Due to the high intrinsic cut-off frequency $(\approx 90 \pm 10$ $\mathrm{GHz}$ ) the present results can also provide the useful advantages of $6 \mathrm{H}-\mathrm{SiC}$ MESFET for high frequency performance.

\section{ACKNOWLEDGEMENT}

I would like to thank Maryam Gholvani for writing up the study. 


\section{REFERENCES}

Agarwal, A.K., 1997. Silicon carbide power device development. Proceedings of the 2nd International All Electric. Electronic Combat Vehicle Conference (AECV-II), June 8-12, Dearborn, MI. USA., pp: 13-19.

Arabshahi, H. and M.H. Ghasemian, 2006. Influence of polarization charges and electron trapping in the buffer layer in wurtzite phase AlGaN/GaN HFETs. Modern Phys. Lett. B, 20: 1397-1 404.

Arabshahi, H., 2006. Monte carlo simulations of electron transport in wurtzite phase gan mesfet including trapping effect. Modern Phys. Lett. B, 20: 787-794.

Dohnke, K., R. Rupp, D. Peters, J. Volkl and D. Stephani, 1994. $6 \mathrm{H}-\mathrm{SiC}$ field effect transistor for high temperature applications. Proceedings of the American Institute of Physics Conference (AIPCS'94), IOP Publishing, Bristol, UK., pp: 625-627.
Joshi, R.P., 1995. Monte carlo calculations of the temperature- and field-dependent electron transport parameters for 4H-SiC. J. Applied Phys., 78: 5518-5521.

Mickevicius, R. and J.H. Zhao, 1998. Monte carlo study of electron transport. J. Applied Phys., 83: 3161-3167.

Nilsson, H.E., U. Sannemo and C.S. Petersson, 1996. Monte Carlo simulation of electron transport in 4H-SiC using a two-band model with multiple minima.

J. Applied Phys., 80: 3365-3369.

Trew, R.J. and M.W. Shin, 1994. Wide bandgap semiconductor MESFETs for high temperature applications. Proceedings of the 3rd International Workshop on Integrated Nonlinear Microwave and Millimeterwave Circuits, Oct. 5-7, Duisburg, Germany, pp: 109-123.

Walmsley, M. and R.A. Abram, 1996. A fast poisson solver for realistic semiconductor device structures. COMPEL: Int. J. Comput. Math. Electr. Electron. Eng., 15: 31-51. 